Virginia Commonwealth University VCU Scholars Compass

2007

\title{
Magnetic endohedral metallofullerenes with floppy interiors
}

Meichun Qian

Virginia Commonwealth University

Stephen V. Ong

Virginia Commonwealth University

Shiv N. Khanna

Virginia Commonwealth University, snkhanna@vcu.edu

Mark B. Knickelbein

Argonne National Laboratory

Follow this and additional works at: http://scholarscompass.vcu.edu/phys_pubs

Part of the Physics Commons

Qian, M., Ong, S.V., Khanna, S.N., et al. Magnetic endohedral metallofullerenes with floppy interiors. Physical Review B, 75, 104424 (2007). Copyright (C) 2007 American Physical Society.

\section{Downloaded from}

http://scholarscompass.vcu.edu/phys_pubs/56

This Article is brought to you for free and open access by the Dept. of Physics at VCU Scholars Compass. It has been accepted for inclusion in Physics Publications by an authorized administrator of VCU Scholars Compass. For more information, please contact libcompass@vcu.edu. 


\title{
Magnetic endohedral metallofullerenes with floppy interiors
}

\author{
Meichun Qian, ${ }^{1}$ Stephen V. Ong, ${ }^{1}$ Shiv N. Khanna, ${ }^{1, *}$ and Mark B. Knickelbein ${ }^{2}$ \\ ${ }^{1}$ Department of Physics, Virginia Commonwealth University, Richmond, Virginia 23284-2000, USA \\ ${ }^{2}$ Chemistry Division and Center for Nanoscale Materials, Argonne National Laboratory, Argonne, Illinois 60439, USA
}

(Received 4 October 2006; published 27 March 2007; corrected 29 May 2007)

\begin{abstract}
It is shown that $\mathrm{Gd}_{3} \mathrm{~N} @ \mathrm{C}_{80}$ is a highly magnetic and very stable motif that allows enhanced contrast magnetic resonance imaging and electric dipole moment with potential for cancer treatment. Using a synergistic approach combining Stern-Gerlach experiments in beams and first-principles electronic structure studies, it is demonstrated that an isolated $\mathrm{Gd}_{3} \mathrm{~N}$ has a ground state spin moment of $23 \mu_{B}$ followed by a noncollinear state of $17.2 \mu_{B}$ only $88 \mathrm{meV}$ above the ground state. The large moment is largely due to localized $f$ electrons. As a $\mathrm{Gd}_{3} \mathrm{~N}$ is embedded inside a $\mathrm{C}_{80}$ cage, the localized $f$ electrons maintain the magnetic character while the hybridization between the $s, d$ states of isolated $\mathrm{Gd}_{3} \mathrm{~N}$ and $p$ states of $\mathrm{C}_{80}$ leads to a strongly bound motif with an interaction energy of $13.63 \mathrm{eV}$ and a large highest-occupied-molecular-orbital-lowest-unoccupiedmolecular-orbital gap of $1.48 \mathrm{eV} . \mathrm{Gd}_{3} \mathrm{~N} @ \mathrm{C}_{80}$ is further shown to possess two isomers corresponding to the location of the $\mathrm{N}$ atom on either side of the $\mathrm{Gd}_{3}$ triangle with an appreciable electric dipole moment and a low barrier of $91 \mathrm{meV}$ for transition between them offering potential for a fluctuating dipole.
\end{abstract}

DOI: 10.1103/PhysRevB.75.104424 PACS number(s): 75.75.+a, 71.20.Tx, 71.15.Mb, 73.22.-f

The possibility of synthesizing fullerene cages with endohedral metal atoms has generated tremendous excitement since the combination can be described as a motif having a metallic core and a covalent outer shell. ${ }^{1,2}$ As the fullerene cage acts to protect the interior metal atoms, it can provide ways of maintaining selected properties of metallic clusters while the endohedral unit is functionalized with ligands or assembled in cluster assemblies. Further, the isolated fullerene cages can be electronically unstable and the metal core can bind strongly to stabilize the cage. This electronic bonding on the one hand can fill the empty electronic levels of the fullerene leading to filled electronic shells and reduced reactivity and, on the other hand, strengthen the unit through covalent/ionic bonding of the metal and cage subunits. The later aspect is important for medical applications since the cages can sometimes open releasing the reactive metal atoms.

One of the systems that have attracted attention in this regard are the fullerenes containing rare earth atoms ${ }^{3}$ and in particular Gadolinium (Gd). An isolated $\mathrm{Gd}$ atom has a large spin-only magnetic moment of $8 \mu_{B}$ where $7 \mu_{B}$ is derived from localized $f$ electrons. When embedded inside the cages, the Gd atom bond through the delocalized s and d electrons, leaving the spin magnetic moment from $f$ electrons, almost intact. $^{4}$ Indeed water-soluble endohedral metallofullerenes ${ }^{5-7}$ functionalized with poly and multihydroxyl groups have been found to be potential contrast enhancing agents for magnetic resonance imaging (MRI). In this regard, a compound that is currently drawing attention is trigadolinium nitride fullerenes ${ }^{8-10} \mathrm{Gd}_{3} \mathrm{~N} @ \mathrm{C}_{80}$. Experiments indicate that it can lead to relaxation enhancement ${ }^{11}$ by as much as a factor of 20 offering the potential for a new class of MRI tumor delineation techniques. The new motifs are also attractive for cancer treatment since one can replace one of the $\mathrm{Gd}$ atoms by $\mathrm{Lu}$ that can be activated by neutrons from outside, leading to radioactive units. ${ }^{12}$ The substitution of $\mathrm{Gd}$ by $\mathrm{Tb}$ can generate fluorescent particles ${ }^{13}$ offering another technique for imaging. In view of such possibilities, these nanoparticles are being considered, in particular, for detecting and treating brain tumors that are hard to access otherwise.

While these are exciting developments leading to novel medical applications of nanoparticles, ${ }^{14}$ a fundamental understanding of the electronic and magnetic properties of such endohedral fullerenes is still lacking and several questions remain unanswered. For example, (1) what are the electronic and magnetic properties of a free $\mathrm{Gd}_{3} \mathrm{~N}$ cluster and how are these modified in going inside the cage? In particular, how large are the magnetic moments at the Gd sites and what is the nature of the magnetic coupling between the Gd moments? How stable is the magnetic state of the unit? (2) What is the strength of interaction between the $\mathrm{Gd}_{3} \mathrm{~N}$ and $\mathrm{C}_{80}$ and how does the bonding between $\mathrm{Gd}_{3} \mathrm{~N}$ and $\mathrm{C}_{80}$ stabilize the endohedral cage? What is the electronic structure of the resulting motif? (3) What features could lead to relaxation enhancements as this may provide a systematic approach to developing better agent? (4) What is the shape of $\mathrm{Gd}_{3} \mathrm{~N}$ and how rigid are these units? Are there features that have not drawn attention previously and that may lead to novel functionalities? An answer to these questions is needed not only to understand and improve the existing assemblies but also to provide information on how functionalities could be tapped to fully exploit these nanoparticles for cancer treatment and other applications.

The purpose of this paper is to offer an in depth study of the electronic structure and magnetic properties of free $\mathrm{Gd}_{3} \mathrm{~N}$ clusters and $\mathrm{Gd}_{3} \mathrm{~N} @ \mathrm{C}_{80}$ to answer some of the above questions and to highlight novel possibilities. We use a synergistic approach combining first principles theoretical studies and Stern-Gerlach experiments on free $\mathrm{Gd}_{3} \mathrm{~N}$ clusters. The theoretical studies are carried out within a density functional framework corrected for the strong correlations with a Hubbard $U$ and employ a scalar relativistic pseudopotential for Gd atoms. The studies indicate that the ground state geometry of a free $\mathrm{Gd}_{3} \mathrm{~N}$ cluster is a pyramidal shaped unit with a total magnetic moment of $23 \mu_{B}$ with collinear spins. A noncollinear state with a spin magnetic moment of $17.2 \mu_{B}$ is only $88 \mathrm{meV}$ above the ground state. Stern-Gerlach experiments on free $\mathrm{Gd}_{3} \mathrm{~N}$ clusters confirm the theoretical findings 
predicting a moment of $17.0 \pm 2.3 \mu_{B}$. When embedded inside the $\mathrm{C}_{80}$ cage, the $\mathrm{Gd}_{3} \mathrm{~N}$ forms bonding states that lead to a high highest-occupied-molecular-orbital(HOMO) - lowestunoccupied-molecular-orbital(LUMO) gap of 1.48 and $1.54 \mathrm{eV}$ for the majority and minority spin channels, respectively. The resulting unit also has almost degenerate collinear and noncollinear states. We further demonstrate floppiness in the ground state. Two states with $\mathrm{N}$ located on opposite sites of the $\mathrm{Gd}_{3}$ plane are degenerate with a small barrier of $91 \mathrm{meV}$ making the transition possible under ordinary conditions. As the cluster has a permanent electric dipole moment, the floppy mode involves a fluctuation in the overall dipole moment. The existence of this mode is confirmed by observed Raman spectra. ${ }^{9}$ Through excitation via an outside oscillating electric field, this may provide alternate ways of treating tumors by hyperthermia.

We start by considering the experimental determination of the magnetic moment of a free $\mathrm{Gd}_{3} \mathrm{~N}$ cluster. These studies employed the Stern-Gerlach magnet where the magnetic clusters in molecular beams are deflected by a gradient magnetic field. The details of the experimental methods have been provided in previous papers. ${ }^{15,16}$ Basically, the experiment was performed using a four-stage, differentially pumped molecular beam deflection apparatus. Gadolinium clusters were produced via pulsed laser vaporization of a cylindrical target of Gd (99.9\%, Rare Metallic Company, Ltd., Tokyo). The gadolinium target was housed in a laser vaporization source through which helium flowed continuously. The laser vaporization source was coupled to a high aspect ratio flow tube $(9 \mathrm{~cm}$ length $\times 0.3 \mathrm{~cm}$ i.d.) whose temperature could be controlled between 55 and $300 \mathrm{~K}$. The residence time of the clusters within the flow tube $(\sim 4 \mathrm{~ms})$ was sufficient to ensure that they were equilibrated to the flow tube temperature prior to expansion into vacuum though a $1.2 \mathrm{~mm}$ diameter orifice at the end of the flow tube. ${ }^{17} \mathrm{Un}$ der these mild expansion conditions, very little supersonic cooling of the clusters' vibronic degrees of freedom is expected ${ }^{18}$ so that the post-expansion cluster temperature is estimated to be close (within $\sim 5 \mathrm{~K}$ ) to that of the flow tube. The expanding jet was skimmed into a molecular beam, which passed through a gradient dipole magnet ${ }^{19}$ capable of producing $B$ fields of up to $\sim 1.2 \mathrm{~T}$ and gradients $(\partial B / \partial z)$ up to $\sim 210 \mathrm{~T} \mathrm{~m}^{-1}$ in the center of the gap. The clusters were then ionized with a spatially expanded $\mathrm{ArF}$ excimer laser $(\lambda=193 \mathrm{~nm}, h \nu=6.4 \mathrm{eV})$, with the resulting singly ionized clusters detected via time-of-flight (TOF) mass spectrometry. The TOF mass spectrometer can be operated either in conventional high-resolution (Wiley-McLaren) mode or in position-sensitive mode. Position-sensitive time-of-flight (PSTOF) mass spectrometry ${ }^{15,20,21}$ allows the spatial distributions of clusters in a molecular beam to be mapped onto the time-domain and thus recorded using a digital oscilloscope. The spatial deflection $\Delta z$ of a cluster ensemble is determined via the positions of the field on vs field off PSTOF peak profiles. Beam deflections as small as $\sim 20 \mu \mathrm{m}$ can be detected by this approach.

The induced magnetization $\left\langle M_{z}\right\rangle$ of ensembles of clusters displaying high field-seeking behavior are calculated from the magnitude of their beam deflection $\Delta z$, as computed from the change in PSTOF mass peak first moments recording with the magnetic field on vs off: $:^{15,22,23}$

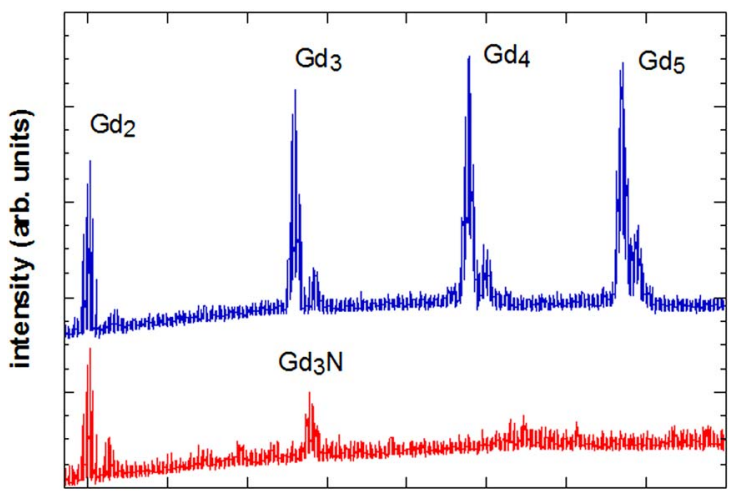

time-of-flight (arb. units)

FIG. 1. (Color online) TOF mass spectra of gadolinium clusters. Top trace: pure helium carrier gas; bottom trace: $0.05 \% \mathrm{~N}_{2}$ added to carrier gas. The small peaks to the high mass side of the $\mathrm{Gd}_{n}$ mass peaks in the top trace are due to the cluster monoxides, $\operatorname{Gd}_{n} \mathrm{O}$.

$$
\left\langle M_{z}\right\rangle=C \Delta z m v^{2}\left(\frac{\partial B}{\partial z}\right)^{-1},
$$

where $m$ is the cluster mass, $v$ is the molecular beam speed, $\partial B / \partial z$ is the field gradient, and $C$ is an apparatus constant. For a superparamagnetic system in thermal equilibrium, and at temperatures $T$ at which the condition $\mu B \ll k T$ holds, as is the case in the present experiment, the intrinsic magnetic moment of the system $\mu$ is related to the measured magnetization by the Curie law ${ }^{22-26}$

$$
\left\langle M_{z}\right\rangle \approx \frac{\mu^{2} B}{3 k T} .
$$

In the present work, high-resolution TOF mass spectra of gadolinium clusters were generated in pure helium and in helium to which a trace $(0.05 \%)$ of molecular nitrogen was added are shown in Fig. 1. The intensities of the bare $\mathrm{Gd}_{n}$ mass peaks for $n \geqslant 3$ are completely depleted in the presence of added nitrogen, indicative of reactions producing $\mathrm{Gd}_{n} \mathrm{~N}_{m}$ species. ${ }^{27} \mathrm{Gd}_{2}$ is considerably less reactive toward nitrogen. Few prominent $\mathrm{Gd}_{3} \mathrm{~N}_{m}$ product peaks are actually observed in the mass spectrum recorded with $\mathrm{N}_{2}$ present, indicating that the product distributions are wide, distributed over many masses and/or that the ionization energies of the products are higher than $6.4 \mathrm{eV}$. As shown in Fig. $1, \mathrm{Gd}_{3}$ is completely depleted even at small $\mathrm{N}_{2}$ concentrations, with $\mathrm{Gd}_{3} \mathrm{~N}$ appearing as the only prominent $\mathrm{Gd}_{3} \mathrm{~N}_{m}$ product. The relatively large intensity of the $\mathrm{Gd}_{3} \mathrm{~N}$ in the mass spectrum compared to other $\mathrm{Gd}_{n} \mathrm{~N}_{m}$ species indicates that it possesses a high thermodynamic stability and/or that it is formed via a kinetically favorable reaction channel.

The PSTOF mass spectrum of $\mathrm{Gd}_{3} \mathrm{~N}$ recorded with the beam deflection off vs on is shown in Fig. 2. The high-field deflection of the $\mathrm{Gd}_{3} \mathrm{~N}$ in the beam, shown by the shift in the corresponding mass peak toward longer arrival times in the mass spectrum, is consistent with superparamagnetic behavior. ${ }^{15,28,29}$ From the magnitude of the observed beam deflection and Eqs. (1) and (2) we calculate an intrinsic moment for $\mathrm{Gd}_{3} \mathrm{~N}$ of $17.0 \pm 2.3 \mu_{B}$. 


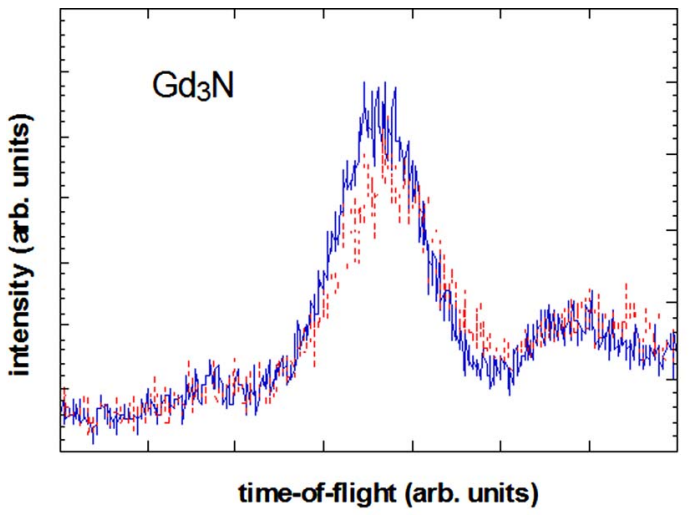

FIG. 2. (Color online) Position-sensitive time-of-flight spectra of $\mathrm{Gd}_{3} \mathrm{~N}$. Solid trace: Deflection magnet off $(B=0 \mathrm{~T}, \partial B / \partial z$ $\left.=0 \mathrm{~T} \mathrm{~m}^{-1}\right)$. Dashed trace: Deflection field on $(B=0.98 \mathrm{~T}, \partial B / \partial z$ $=192 \mathrm{~T} \mathrm{~m}^{-1}$ ).

The theoretical studies were carried out using the Vienna $A b$ initio simulation package (VASP) based on the density functional theory. ${ }^{30}$ The exchange and correlation potentials were treated using the PBE form ${ }^{31}$ for the generalized gradient approximation (GGA). Since the correlation effects are important to properly treat $\mathrm{Gd} f$ states, a $\mathrm{LDA}+U$ method $^{32}$ was adopted in order to separate the occupied $4 f$ orbitals from the unoccupied ones. The Hubbard parameter $U$ $=8 \mathrm{eV}$ was chosen for the effective Coulomb interaction ${ }^{33}$ and a value of $0.8 \mathrm{eV}$ for the exchange parameter on the $\mathrm{Gd}$ ion was used. A plane-wave basis set and the projector augmented wave (PAW) pseudopotentials for $\mathrm{Gd}, \mathrm{N}$, and $\mathrm{C}$ elements with 18,5 , and 4 valence electrons, respectively, were employed. Note that the $4 f$ electrons are included in the valence shell. The energy cutoff was set to $300 \mathrm{eV}$. The convergence in energy was set as $1 \mathrm{meV}$.

The actual calculations were carried out using a cubic supercell with the lattice constant of $20 \AA$. The large size of the unit cell ensures a minimal interaction between the clusters in the neighboring cells when using the periodic boundary conditions. We took the $\Gamma$ point to sample the first Brillouin zone. The Wigner-Seitz sphere radii of $\mathrm{Gd}, \mathrm{N}$, and $\mathrm{C}$ atoms were chosen as $1.79,0.95$, and $0.92 \AA$, respectively, to construct the projected densities of states. All the geometries were fully optimized using the criteria that the force on each atom is less than $1 \mathrm{meV} / \AA$.

In order to establish the accuracy of our theoretical approach, we start by discussing the electronic properties and spin multiplicity of a free $\mathrm{Gd}$ atom and a $\mathrm{Gd}_{2}$ for which the experimental results are available. For a single Gd atom [Fig. 3(a)], our studies predict an ionization potential of $6.02 \mathrm{eV}$ and a spin multiplicity of 9 compared to the experimental values of 6.15 and $9 \mathrm{eV}$, respectively. The $\mathrm{Gd}_{2}$ is experimentally known to be highest moment per atom ${ }^{34}$ with a spin multiplicity of 19 . Our studies on $\mathrm{Gd}_{2}$ indeed find a spin multiplicity of 19 [see Fig. 3(b)]. Further, our calculations yield a bond length of $2.92 \AA$ in good agreement with the experimental value of $2.895 \AA .{ }^{34}$ However, the binding energy is not known experimentally. The close agreement indicates that the present approach is well placed to determine the electronic and magnetic properties of these systems. This
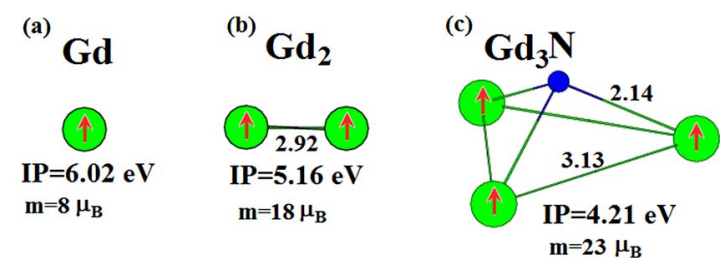

(d)
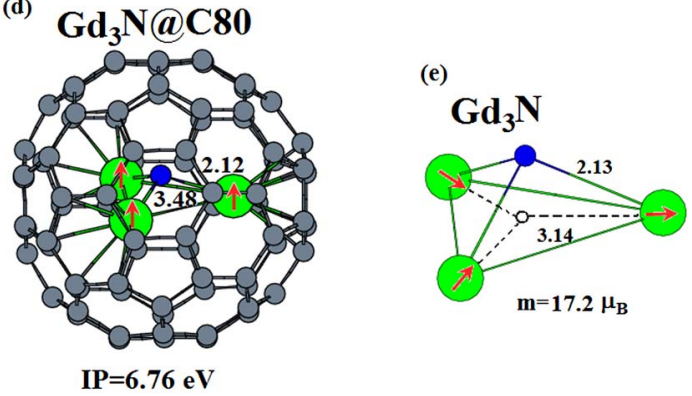

FIG. 3. (Color online) The equilibrium geometries of the Gadolinium clusters for the collinear ferromagnetic configurations and noncollinear magnetic configuration. The total spin magnetic moment is also given (in unit of $\mu_{B}$ ).

is further confirmed by our studies on $\mathrm{Gd}_{3} \mathrm{~N}$.

For a free $\mathrm{Gd}_{3} \mathrm{~N}$ cluster, the planar and non-planar configurations were examined. Further, within a collinear magnetic picture, the magnetic moments at the Gd sites could be ferromagnetically coupled or one of the Gd moments could be antiferromagnetically aligned to the other two moments. In each case, the spin moment needs to be determined. Theoretical studies exploring all these possibilities were carried out and the ground state was determined to be a pyramidal configuration shown in Fig. 3(c). Note that the cluster has a spin magnetic moment of $23 \mu_{B}$ arising primarily due to ferromagnetically coupled moments of $4 f$ electrons on each of the Gd atom. While this is interesting, previous studies on pure $\mathrm{Gd}_{n}$ clusters have shown that the spin moments can assume canted configuration. ${ }^{35}$ This is the reason that the spin magnetic moment per atom, measured via Stern-Gerlach experiments on free $\mathrm{Gd}_{n}$ clusters, are found to be much smaller than the intrinsic moments on each Gd atom. ${ }^{36}$ It is therefore important to explore the possibility of such canting effects in the present case. These were investigated via a noncollinear (NCL) spin calculations performed within a projector augmented wave function formalism as implemented ${ }^{37}$ with VASP. We indeed find a noncollinear configuration [as shown in Fig. 3(e)] with a spin magnetic moment of all $17.2 \mu_{B}$ only $88 \mathrm{meV}$ above the ground state. The small energy difference is within the accuracy of the calculations and indicates that the canted state may be accessible under ordinary conditions. It is interesting to note that experimental measured moment of $17.0 \pm 2.3 \mu_{B}$ lies in the range $17.2-23 \mu_{B}$ indicating that the spin canted state is experimentally accessible.

The energy difference between the collinear ferromagnetic and the antiferromagnetic states can be mapped to a Heisenberg Hamiltonian to derive the strength of the exchange coupling. To accomplish this, we assumed a Heisenberg model Hamiltonian with an exchange coupling parameter $J$, namely, 


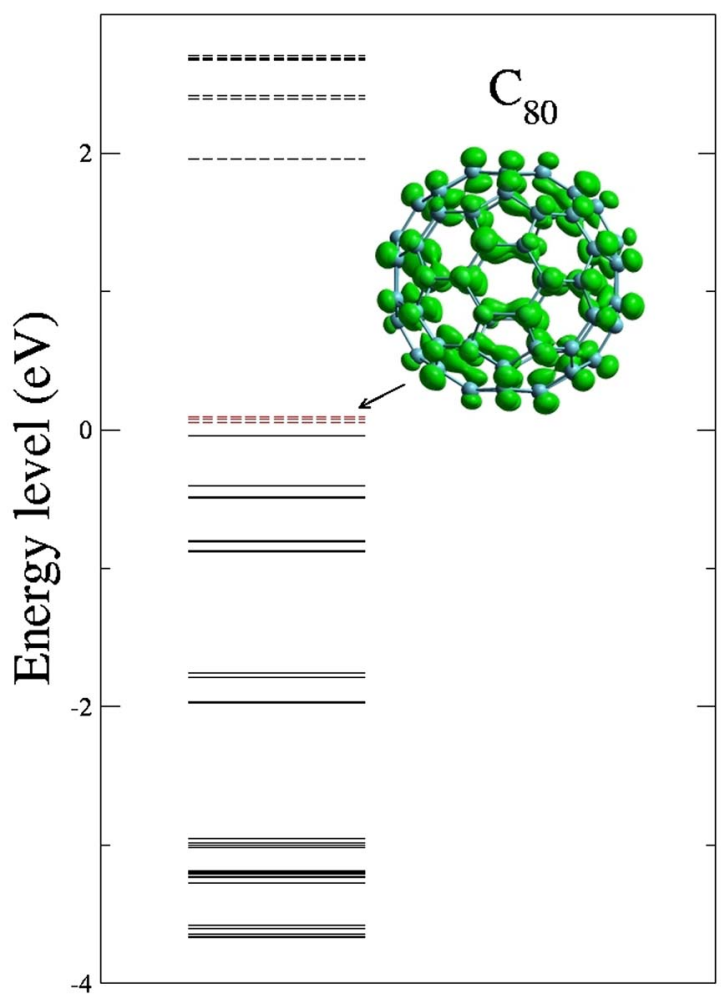

FIG. 4. (Color online) One electron energy levels for the fullerene $\mathrm{C}_{80}$. The three LUMO orbitals are indicated by red lines and their charge density surrounding the cage is shown by the green isosurface with the value of 0.03 electrons per $\AA^{3}$.

$$
H=-\sum_{i, j(\neq i)} J S_{i} S_{j}
$$

Here $S_{i}$ and $S_{j}$ are the spin magnetic moments on the Gd sites $i$ and $j$, respectively. We assumed the same exchange parameter $J$ between three $\mathrm{Gd}$ atomic sites and the same spin moment $S=7 / 2$. The energy difference between the antiferromagnetic and ferromagnetic orders is then

$$
E_{\mathrm{FA}}=E_{\mathrm{AFM}}-E_{\mathrm{FM}}=4 J S^{2}
$$

and was fitted to the calculated energies for various spin states to determine an effective exchange parameter $J$. Our studies give $J=4.63 \mathrm{meV}$ indicating an appreciable magnetic coupling.

The next issue is whether the magnetic coupling in the free cluster is maintained when it is embedded inside the $\mathrm{C}_{80}$ cage (see Fig. 4). It is important to first examine the electronic properties of an isolated $\mathrm{C}_{80}$ cage. As before, the $\mathrm{C}_{80}$ cluster was placed inside a cube of side $20 \AA$. The shortest distance between the $\mathrm{C}$ atoms in the neighboring supercells is $11.9 \AA$ ensuring a negligible interaction between the $\mathrm{C}_{80}$ clusters in neighboring cells. The $\mathrm{C}$ - $\mathrm{C}$ distance in the optimized cluster range from 1.42 to $1.45 \AA$. It has an ionization potential of $4.83 \mathrm{eV}$ and an electron affinity of $3.85 \mathrm{eV}$. The large electron affinity results from the unfilled states close to the highest occupied molecular orbital. This is easily seen in Fig. 4, which shows the ground state geometry and the one electron levels. The continuous lines correspond to the occu- pied levels while the dotted lines represent the unoccupied states. Note that the cluster is characterized by three unoccupied levels that can accommodate up to six electrons. This shows that endohedral species that can lead to the filling of the available states can bind strongly and also stabilize the complex. Can $\mathrm{Gd}_{3} \mathrm{~N}$ accomplish it while maintaining its magnetic character?

To examine the location of the $\mathrm{Gd}_{3} \mathrm{~N}$, several initial configurations were tried. The Gd atoms could bond with pentagonal or hexagonal faces. Keeping this in mind, two symmetric possibilities corresponding to the three Gd atoms all bound to the hexagonal or to the pentagonal rings on the surface were explored. In each case, the geometry was optimized without any symmetry constraint and various spin multiplicities including the possibility of antiferromagnetic coupling were tried. The ground state configuration shown in Fig. 3(d) corresponds to the Gd atoms bound to the hexagonal rings. The $\mathrm{N}$ atom is again bound to the three $\mathrm{Gd}$ atoms leading to a pyramidal shaped $\mathrm{Gd}_{3} \mathrm{~N}$ as in case of free cluster with the exception that the Gd-Gd bonds are slightly stretched from their value for the free cluster. Further, the Gd-C distance is $2.49 \AA$ and there is a strong interaction between the Gd $d$ and C $p$ states. The binding energy of the $\mathrm{Gd}_{3} \mathrm{~N}$ to the cluster was calculated using the equation

$$
\mathrm{BE}=E\left(\mathrm{Gd}_{3} \mathrm{~N}\right)+E\left(\mathrm{C}_{80}\right)-E\left(\mathrm{Gd}_{3} \mathrm{~N} @ \mathrm{C}_{80}\right),
$$

where $E\left(\mathrm{Gd}_{3} \mathrm{~N}\right), E\left(\mathrm{C}_{80}\right)$, and $E\left(\mathrm{Gd}_{3} \mathrm{~N} @ \mathrm{C}_{80}\right)$ are the total energies of the $\mathrm{Gd}_{3} \mathrm{~N}$, isolated $\mathrm{C}_{80}$ and $\mathrm{Gd}_{3} \mathrm{~N} @ \mathrm{C}_{80}$ clusters, respectively. The calculated binding energy is $13.63 \mathrm{eV}$. The cluster has an ionization potential of $6.76 \mathrm{eV}$ and an electron affinity of $2.51 \mathrm{eV}$. The large binding energy and the reduction of the electron affinity from that in an isolated $\mathrm{C}_{80}$ are indicative of the strong interaction between $\mathrm{Gd}_{3} \mathrm{~N}$ and $\mathrm{C}_{80}$ states, and filling of the lowest empty $\mathrm{C}_{80}$ levels. A Bader atomic charge analysis, ${ }^{38}$ indicates that around 3.66 electrons are transferred from the molecule $\mathrm{Gd}_{3} \mathrm{~N}$ to the $\mathrm{C}_{80}$ cage. Figure 5(a) shows the Bader charge volume with the isosurface of 0.7 electron per $\AA^{3}$, which indicates the bonding directions between the $\mathrm{Gd}$ and the carbons of hexagonal rings. Does the large magnetic moment of the free $\mathrm{Gd}_{3} \mathrm{~N}$ survive during the strong interaction?

The resulting cluster had two almost degenerate magnetic states namely a collinear configuration with a spin magnetic moment of $21 \mu_{B}$ and a noncollinear state corresponding to a spin moment of $14.1 \mu_{B}$. In fact, the noncollinear state is around $10 \mathrm{meV}$ more stable. An analysis of the electronic orbitals shows that the large moment is associated with $f$ electrons of the rare earth atom. The $f$ states on each $\mathrm{Gd}$ atom contribute $7 \mu_{B}$ indicative of that the localized $f$ states maintain their atomic spin moment. This brings out an important partitioning of the electronic levels. The $s$ and $d$ states of $\mathrm{Gd}$ and $p$ states of $\mathrm{N}$ hybridize with the $s, p$ states of the $\mathrm{C}$ atoms in the spherical cage to create strong bonds that stabilize the caged motif while the protected $f$ states maintain the magnetic character. To see this more clearly, we show in Fig. 5(b) the one electron levels in $\mathrm{Gd}_{3} \mathrm{~N} @ \mathrm{C}_{80}$. Also shown are the orbital projected density of states obtained by broadening each level with a Gaussian of width $0.05 \mathrm{eV}$ and then projecting on to the $\operatorname{Gd} s, \operatorname{Gd} d$, and $\mathrm{N} p$ states. Two 


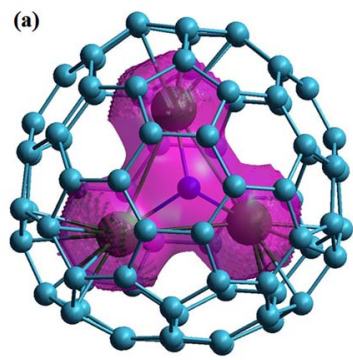

(b)

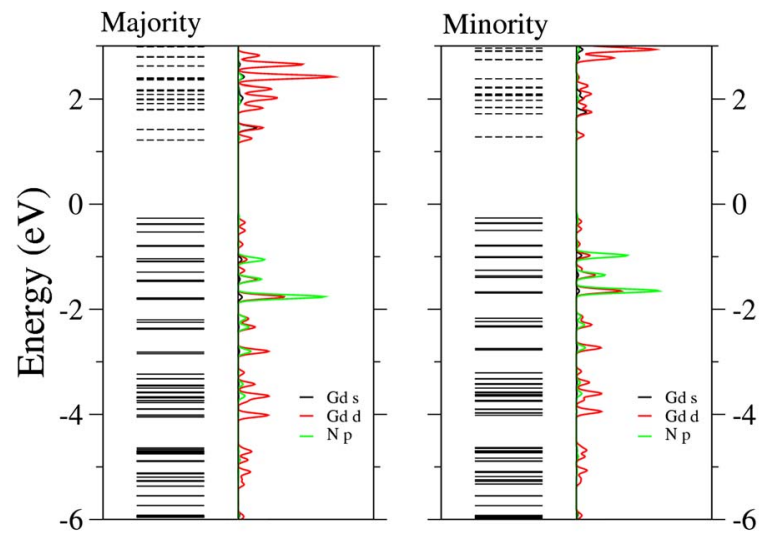

FIG. 5. (Color online) The isosurface of the Bader charge volume of the molecule with the value of 0.7 electrons per $\AA^{3}$ as the molecule $\mathrm{Gd}_{3} \mathrm{~N}$ is embedded in the fullerene $\mathrm{C}_{80}$ cage. (b) One electron energy levels in spin majority and minority channels for the $\mathrm{Gd}_{3} \mathrm{~N}$ embedded in the $\mathrm{C}_{80}$ cage. The densities of states are projected on the $\mathrm{N}$ and $\mathrm{Gd}$ atomic sites.

features draw attention. First, there is a large HOMO-LUMO gap of $1.48 \mathrm{eV}$, which is in good agreement with the photoemission experimental gap 9 of $1.75 \mathrm{eV}$, and that the empty states near the HOMO in the bare $\mathrm{C}_{80}$ are now filled. Secondly, the projected spin density of states for the two spin states are similar showing no net spin polarization. The Gd $d$ density of states are, however, dissimilar in the excited region. Since the $\mathrm{Gd} d$ states hybridize with the $\mathrm{C} s, p$ states, this suggests that it may be possible to have net spin polarization on the cage by occupying the excited states. This aspect is particularly important for magnetic resonance imaging since a net polarization located on the cage can affect resolution. This will form the basis of another paper.

The particular geometry of $\mathrm{Gd}_{3} \mathrm{~N} @ \mathrm{C}_{80}$ raises an interesting possibility. As $\mathrm{Gd}_{3} \mathrm{~N}$ has a nonplanar geometry, is it possible to have other isomers? We indeed found that the motif is characterized by another isoenergetic configuration that corresponds to $\mathrm{N}$ atoms located on the other side of the $\mathrm{Gd}_{3}$ triangle (see Fig. 6). To further probe the nature of the two isomers and to explore if it may be possible to hop between the two minima, we calculated the barrier for the transition. The $\mathrm{N}$ atom was maintained at different heights above the $\mathrm{Gd}_{3}$ plane and the geometry was optimized by freezing the height. Figure 6 shows the energy as a function of height. It is truly remarkable that the barrier is only $91 \mathrm{meV}$. It makes it possible for the $\mathrm{N}$ atoms to hop between the two isomeric states under ordinary conditions. By studying the curvature of the energy curve, we also calculated the vibrational fre-

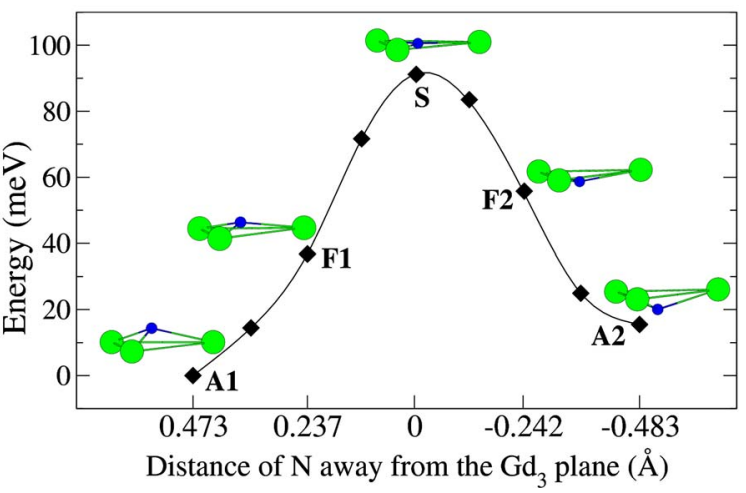

FIG. 6. (Color online) Inside the $\mathrm{C}_{80}$ cage, the molecule $\mathrm{Gd}_{3} \mathrm{~N}$ has the energy barrier for the $\mathrm{N}$ atom moving through the $\mathrm{Gd}_{3}$ plane. The labels correspond to the configurations along the path. $\mathrm{A} 1$ and $\mathrm{A} 2$ are two mirror configurations and $\mathrm{S}$ is the transition state.

quency for the $\mathrm{N}$ atom. It has a value of $85 \mathrm{~cm}^{-1}$. It is remarkable to note that this frequency matches the low frequency mode in the observed Raman spectra 9 confirming the theoretical finding. The occurrence of this low frequency modes indicates that materials assembled using such motifs may exhibit extraordinary attributes. For example, our studies show that the cluster has a net dipole moment of $0.71 \mathrm{~d}$ largely associated with the charges localized at $\mathrm{N}$ and $\mathrm{Gd}_{3}$. It may then be possible to absorb energy into the system by irradiating with the external radiation. This would heat the clusters and hence may provide an alternate possibility of killing the cancer region through local heating. To our knowledge, this possibility has not been explored before.

To summarize, the present study shows that the endohedral metallofullerene $\mathrm{Gd}_{3} \mathrm{~N} @ \mathrm{C}_{80}$ is a highly stable species where the $\mathrm{Gd}_{3} \mathrm{~N}$ cluster is bound to the $\mathrm{C}_{80}$ cage by $13.63 \mathrm{eV}$. The large binding energy makes it unlikely for the cluster to break and release $\mathrm{Gd}$ atoms. The cluster has a filled valence shell that is separated by $1.48 \mathrm{eV}$ from the LUMO and hence is not highly reactive. Despite a closed electronic shell, the cluster is highly magnetic subunit with a high magnetic moment $21 \mu_{B}$ that is largely localized on the $f$ electrons of the $\mathrm{Gd}$ atoms. While there is no appreciable spin density on the cage, if the excited states could be populated, one should be able to generate a net spin density. This is probably the reason that only the endohedral fullerenes coated with hydroxyl groups are found to exhibit high relaxation. We also show that the cluster has a net electric dipole moment that could be inverted with a very small barrier. Using radiation of appropriate frequency, it may be possible to heat these fullerenes thus offering alternate potential for treatments.

M.Q., S.V.O., and S.N.K. acknowledge support from National Institute of Health Grant No. R01-CA119371-01. The experimental portion of this work is supported by the US Department of Energy, Office of Basic Energy Sciences, Division of Chemical Sciences, under Contract W-31-109ENG-38 and by the CREST (Core Research for Evolutional Science and Technology) program of the Japan Science and Technology Agency (JST). S.N.K. is grateful to VCU for providing a study/research leave. 
*Email address: snkhanna@vcu.edu

${ }^{1}$ H. Shinohara, Rep. Prog. Phys. 63, 843 (2000).

${ }^{2}$ Endofullerenes: A New Family of Carbon Clusters, edited by T. Akasaka and S. Nagase (Kluwer Academic Publishers, Dordrecht, 2002).

${ }^{3}$ H. Funasaka, K. Sakurai, Y. Oda, K. Yamamoto, and T. Takahashi, Chem. Phys. Lett. 232, 273 (1995).

${ }^{4}$ L. Senapati, J. Schrier, and K. B. Whaley, Nano Lett. 4, 2073 (2004).

${ }^{5}$ M. Mikawa, H. Kato, M. Okumura, M. Narataki, Y. Kanazawa, N. Miwa, and H. Shinohara, Bioconjugate Chem. 12, 510 (2001).

${ }^{6}$ H. Kato, Y. Kanazawa, M. Okumura, A. Taninaka, T. Yokawa, and H. Shinohara, J. Am. Chem. Soc. 125, 4391 (2003).

${ }^{7}$ R. D. Bolskar, A. F. Benedetto, L. O. Husebo, R. E. Price, E. F. Jackson, S. Wallace, L. J. Wilson, and J. M. Alford, J. Am. Chem. Soc. 125, 5471 (2003).

${ }^{8}$ S. Stevenson, J. P. Phillips, J. E. Reid, M. M. Olmstead, S. P. Rath, and A. L. Balch, Chem. Commun. (Cambridge) 2004, 2814.

${ }^{9}$ M. Krause and L. Dunsch, Angew. Chem., Int. Ed. 44, 1557 (2005).

${ }^{10}$ S. Stevenson, R. R. Stephen, T. M. Amos, V. R. Cadorette, J. E. Reid, and J. P. Phillips, J. Am. Chem. Soc. 127, 12776 (2005).

${ }^{11}$ P. P. Fatouros, F. D. Corwin, Z-J Chen, W. C. Broaddus, J. L. Tatum, B. Kettenmann, Z. Ge, H. W. Gibson, J. L. Russ, A. P. Leonard, J. C. Duchamp, and H. C. Dorn, Radiology 240, 756 (2006)

${ }^{12}$ E. B. Iezzi, J. C. Duchamp, K. R. Fletcher, T. E. Glass, and H. C. Dorn, Nano Lett. 2, 1187 (2002).

${ }^{13}$ L. Dunsch, M. Krause, J. Noack, and P. Georgi, J. Phys. Chem. Solids 65, 309 (2004).

${ }^{14}$ L. J. Wilson, D. W. Cagle, T. P. Thrash, S. J. Kennel, S. Mirzadeh, J. M. Alford, and G. J. Ehrhardt, Coord. Chem. Rev. 190-192, 199 (1999).

${ }^{15}$ M. B. Knickelbein, J. Chem. Phys. 116, 9703 (2002).

${ }^{16}$ M. B. Knickelbein, Phys. Rev. B 70, 014424 (2004).

${ }^{17}$ M. B. Knickelbein, S. Yang, and S. J. Riley, J. Chem. Phys. 93,
$94(1990)$

${ }^{18}$ B. A. Collings, A. Amrein, D. M. Rayner, and P. A. Hackett, J. Chem. Phys. 99, 4174 (1993).

${ }^{19}$ D. McColm, Rev. Sci. Instrum. 37, 1115 (1966).

${ }^{20}$ W. A. de Heer and P. Milani, Rev. Sci. Instrum. 62, 670 (1991).

${ }^{21}$ J. L. Persson, Ph.D. thesis, University of California, Los Angeles, 1991.

${ }^{22}$ J. A. Becker and W. A. de Heer, Ber. Bunsenges. Phys. Chem. 96, 1237 (1992).

${ }^{23}$ J. P. Bucher and L. A. Bloomfield, Int. J. Mod. Phys. B 7, 1079 (1993).

${ }^{24}$ S. N. Khanna and S. Linderoth, Phys. Rev. Lett. 67, 742 (1991).

${ }^{25}$ A. Châtelain, Philos. Mag. B 79, 1367 (1999).

${ }^{26}$ M. B. Knickelbein, J. Chem. Phys. 121, 5281 (2004).

${ }^{27}$ M. B. Knickelbein, Annu. Rev. Phys. Chem. 50, 79 (1999).

${ }^{28}$ M. B. Knickelbein, Phys. Rev. Lett. 86, 5255 (2001).

${ }^{29}$ M. B. Knickelbein, Phys. Rev. B 71, 184442 (2005).

${ }^{30}$ G. Kresse and J. Hafner, J. Phys.: Condens. Matter 6, 8245 (1994); G. Kresse and J. Furthmüller, Phys. Rev. B 54, 11169 (1996).

${ }^{31}$ J. P. Perdew, K. Burke, and M. Ernzerhof, Phys. Rev. Lett. 77, 3865 (1996).

${ }^{32}$ A. I. Liechtenstein, V. I. Anisimov, and J. Zaanen, Phys. Rev. B 52, R5467 (1995); V. I. Anisimov, F. Aryasetiawan, and A. I. Lichtenstein, J. Phys.: Condens. Matter 9, 767 (1997).

${ }^{33}$ M. D. Johannes and W. E. Pickett, Phys. Rev. B 72, 195116 (2005).

${ }^{34}$ R. J. Zee, S. Li, and W. Weltner, J. Chem. Phys. 100, 4010 (1994).

${ }^{35}$ D. P. Pappas, A. P. Popov, A. N. Anisimov, B. V. Reddy, and S. N. Khanna, Phys. Rev. Lett. 76, 4332 (1996).

${ }^{36}$ D. Gerion, A. Hirt, and A. Chatelain, Phys. Rev. Lett. 83, 532 (1999).

${ }^{37}$ D. Hobbs, G. Kresse, and J. Hafner, Phys. Rev. B 62, 11556 (2000).

${ }^{38}$ G. Henkelman, A. Arnaldsson, and A. Jónsson, Comput. Mater. Sci. 36, 354 (2006) 